\title{
Medical renal diseases are frequent but often unrecognized in adult autopsies
}

\author{
Marie E Perrone ${ }^{1}$, Anthony Chang ${ }^{2}$ and Kammi J Henriksen ${ }^{2}$ \\ ${ }^{1}$ Department of Pathology, The University of Washington, Seattle, WA, USA and ${ }^{2}$ Department of Pathology, \\ The University of Chicago, Chicago, IL, USA
}

\begin{abstract}
Kidney diseases affect many hospitalized patients and contribute to morbidity and mortality. Therefore, kidney disease should be prevalent, but the frequency and spectrum of medical renal pathology in autopsy specimens has not been well documented. We sought to determine the spectrum of medical renal pathology in adult autopsy specimens and the frequency of overlooked diagnoses. We reviewed the hematoxylin- and eosin-stained kidney sections from 140 adult autopsies performed at a large teaching hospital over a 2-year period. Fifty-eight cases (41\%) had findings warranting further analysis, including alterations in glomerular matrix and/or cellularity, atypical or pigmented casts, thrombi, tubulointerstitial or vascular inflammation, or deposition of amorphous material. After additional studies and clinical correlation, the pathologic changes in 43 cases (31\%) were categorized as follows: diabetic nephropathy, bile cast nephropathy, thrombotic microangiopathy, infectionrelated glomerulonephritis, focal necrotizing/crescentic glomerulonephritis, oxalate nephropathy, light-chain cast nephropathy, amyloidosis, urate nephropathy, hemosiderosis, antineutrophil cytoplasmic antibodyassociated vasculitis, polyoma virus nephropathy, atheroembolic disease, and nephrocalcinosis. These diagnoses were not reported in $26(60 \%)$ cases during the initial autopsy evaluation. This study demonstrates that medical renal diseases are common in autopsy cases, but significant diagnoses can be easily overlooked. Autopsy kidney specimens are a rich source of renal pathology and their evaluation should be emphasized in anatomic pathology residency training. Ultimately, our understanding of how kidney disease contributes to morbidity and mortality will benefit from accurate recognition of renal pathology in autopsy specimens.
\end{abstract}

Modern Pathology (2018) 31, 365-373; doi:10.1038/modpathol.2017.122; published online 6 October 2017

Kidney disease is the ninth leading cause of death in the United States, accounting for more than 50000 deaths annually. ${ }^{1}$ Chronic kidney disease and endstage renal disease also substantially increase the risks of cardiovascular disease and death. ${ }^{2}$ More than 650000 Americans have end-stage renal disease, and the prevalence of chronic kidney disease in the United States was estimated at $13.6 \%$ in $2012 .^{3}$ Additionally, acute kidney injury occurs in over $22 \%$ of hospitalizations and is associated with a more than fourfold increased mortality risk. ${ }^{4}$ For these reasons, we expect a high rate of acute and chronic kidney disease in medical autopsies. There have been numerous autopsy series detailing renal pathology findings in particular cohorts, including patients with hematopoietic stem cell transplants, ${ }^{5,6}$ HIV/AIDS, ${ }^{7-10}$ hematologic malignancy, ${ }^{11,12}$ hepatitis

Correspondence: Dr KJ Henriksen, MD, Department of Pathology, The University of Chicago, 5841 South Maryland Avenue, Room S-630 (MC 6101), Chicago, IL 60637, USA.

E-mail: kammi.henriksen@uchospitals.edu

Received 9 May 2017; revised 27 July 2017; accepted 1 August 2017; published online 6 October 2017
$\mathrm{C},{ }^{13,14}$ diabetes, ${ }^{15}$ severe liver dysfunction, ${ }^{16}$ illicit drug abuse, ${ }^{17}$ disseminated intravascular coagulation, ${ }^{18}$ IgG4-related kidney disease, ${ }^{19}$ scleroderma, ${ }^{20}$ leprosy, ${ }^{21}$ cancer, ${ }^{22}$ and myocardial infarct. ${ }^{23}$ In addition, there was one large autopsy study from Japan focusing on lesions related to hypertension and diabetes, which found that death was directly attributable to renal disease in $2.2 \%$ of cases, ${ }^{24}$ and one recent Turkish study documenting frequent immune complex deposition (namely IgA) in autopsy kidneys. ${ }^{25}$ However, the full incidence and spectrum of medical renal disease at autopsy has not been well described.

Most medical autopsies are conducted and reported by either general surgical pathologists or pathologists subspecialized in areas other than medical renal pathology. In recent years, it has been reported that non-neoplastic kidney diseases are often missed in tumor nephrectomy specimens, likely due to suboptimal training in medical renal pathology. ${ }^{26-30}$ For these reasons, we speculate that renal pathology might be easily overlooked during the histologic examination of autopsy kidneys. The purpose of this study is to establish the baseline incidence and spectrum of medical renal pathology 
seen in adult autopsies, and to determine the prevalence of unrecognized diagnoses.

\section{Materials and Methods}

We reviewed the autopsy records at the University of Chicago Department of Pathology from January 2013 through December 2014 following approval by the Institutional Review Board, and identified 205 adult autopsies. Exclusion criteria included pediatric autopsies, renal allografts, and specimens with findings of end-stage renal disease. The kidneys had been evaluated by a fellowship-trained renal pathologist in 65 (32\%) of the 205 autopsy cases, so these cases were analyzed separately. These included 43 cases in which the renal pathologist was consulted by the primary autopsy pathologist due to abnormal histologic findings and/or clinical history of kidney disease, and 22 cases in which the renal pathologist performed the autopsy. For the remaining 140 adult autopsies, the hematoxylin and eosin (H\&E)-stained kidney slides were evaluated by two observers (MEP and $\mathrm{KJH}$ ) without the knowledge of clinical information.

Identification of histologic alterations triggered subsequent evaluation by periodic acid-Schiff (PAS) staining. Jones methenamine silver stains were examined in a subset of cases with thrombi and/or duplication of the glomerular basement membranes. Congo red stains were performed in a number of cases with mesangial sclerosis or deposition of amorphous material. Prussian blue stains were examined in cases with prominent pigment accumulation in the tubular epithelial cells. Immunofluorescence microscopy for $\operatorname{IgG}$, IgA, IgM, and $\kappa$ and $\lambda$ light chains was performed on the paraffin tissue sections in cases with histologic alterations including glomerular hypercellularity (endocapillary and/or mesangial), glomerular basement membrane thickening and/or duplication, and atypical intratubular casts. ${ }^{31}$ Briefly, $4 \mu \mathrm{m}$ sections were cut from the original paraffin blocks, deparaffinized with $0.25 \%$ trypsin-ethylenediaminetetraacetic acid for $1.5 \mathrm{~h}$ at $37^{\circ} \mathrm{C}$, washed, and placed in phosphatebuffered saline. They were subsequently stained with fluorescein isothiocyanate-conjugated antibodies to IgG, IgA, IgM, or $\kappa$ or $\lambda$ light chains (DAKO, Carpinteria, CA, USA) for 1 to $3 \mathrm{~h}$. Standard immunohistochemistry techniques were used when indicated, including SV40 (Calbiochem EMD Biosciences, San Diego, CA, USA), CD61 (DAKO), myoglobin (DAKO), and hemoglobin A (DAKO). Clinical correlation was performed. We reviewed the original autopsy reports to determine whether the renal lesions were initially identified, and any discrepancies were noted. Owing to difficulty differentiating between acute tubular injury/'necrosis' and post-mortem autolysis based on histology alone, these two processes were disregarded. ${ }^{32}$ Additionally, the following pathologic parameters of global glomerulosclerosis, interstitial fibrosis, tubular atrophy, and arteriosclerosis were not used to determine whether discrepancies occurred during the initial evaluation. Diabetic nephropathy was classified as severe if nodular glomerulosclerosis was present and involving $>50 \%$ of the glomeruli; the remaining cases were classified as mild to moderate.

\section{Results}

A total of 140 autopsies during the designated 2-year span met the inclusion criteria for this study, including 64 (46\%) male and 76 (54\%) female patients (average age 61 years, range 18-92 years). Patient demographics and the documented immediate cause of death are summarized in Table 1.

We identified $58(41 \%)$ cases with histologic alterations that warranted additional studies. Glomerular findings that provoked further evaluation included diffuse and/or nodular mesangial sclerosis, mesangial hypercellularity (defined as more than 3 cell nuclei per peripheral mesangial area on a $4-\mu \mathrm{m}-$ thick tissue section), endocapillary hypercellularity, glomerular basement membrane thickening and/or duplication, and thrombi. Notable tubulointerstitial alterations included interstitial inflammation, cytologic atypia in the tubular epithelial cells, atypical or pigmented casts, and crystals. Pathologic vascular findings included vasculitis, thrombi, and mural deposition of amorphous material. Fifteen cases failed to show a definitive pathologic diagnosis. The remaining $43(31 \%)$ cases showed medical renal diseases categorized as follows (some with multiple diagnoses): diabetic nephropathy, bile cast nephropathy, thrombotic microangiopathy, infection-related glomerulonephritis, focal necrotizing/crescentic glomerulonephritis, light-chain cast

Table 1 Patient demographics and immediate cause of death in 140 reviewed adult autopsies

\begin{tabular}{lc}
\hline Patient variable & $\mathrm{N}=140$ \\
\hline Age & $61(18-92)$ \\
Gender & \\
Male & $64(46 \%)$ \\
Female & $76(54 \%)$ \\
& \\
Race/ethnicity & \\
Black/African American & $85(61 \%)$ \\
Caucasian & $38(27 \%)$ \\
Hispanic/Latino & $5(4 \%)$ \\
Other/unknown & $12(8 \%)$ \\
& \\
Immediate cause of death & $36(26 \%)$ \\
Cardiovascular & $31(22 \%)$ \\
Infection/sepsis & $28(20 \%)$ \\
Malignancy & $13(9 \%)$ \\
Neurologic injury/stroke & $3(2 \%)$ \\
Interstitial lung disease & $3(2 \%)$ \\
Decompensated cirrhosis & $26(19 \%)$ \\
Multifactorial/other/unknown &
\end{tabular}


nephropathy, amyloidosis, oxalate nephropathy, urate nephropathy, hemosiderosis, antineutrophil cytoplasmic antibody (ANCA)-associated vasculitis, polyoma virus nephropathy, atheroembolic disease, and nephrocalcinosis. At least one significant pathologic diagnosis was overlooked in 26 of the 43 cases $(60 \%)$ during the initial autopsy evaluation, detailed in Table 2. Notably, the kidney histology in 23 of the 140 total cases $(16 \%)$ was originally described as 'autolysis' precluding pathologic evaluation at the time of autopsy. Upon further review, 22 were deemed interpretable and 4 had significant missed diagnoses ( 2 cases of diabetic nephropathy, 1 case of light-chain cast nephropathy, and 1 case of thrombotic microangiopathy).

Diabetic nephropathy was identified in 22 (16\%) cases, characterized by glomerular enlargement, mesangial sclerosis, basement membrane thickening, and arteriolar hyalinosis. Eleven were classified as mild-to-moderate diabetic nephropathy with diffuse mesangial sclerosis, and 11 cases as severe diabetic nephropathy with nodular mesangial sclerosis (Figure 1). Review of medical records confirmed that all patients had a history of either type 1 or 2 diabetes mellitus. The 11 cases of mild-to-moderate diabetic nephropathy were all missed at the initial autopsy evaluation, whereas the 11 cases of severe diabetic nephropathy with prominent KimmelstielWilson nodules had been identified by the primary pathologist. Several cases with nodular mesangial sclerosis had accompanying negative Congo red stains, excluding amyloidosis. One diabetic patient also had widespread evidence of recent atheroembolic disease, in which numerous arcuate and interlobular arteries were occluded by cholesterol clefts associated with entrapped fibrin and red blood

Table 2 Diagnoses and frequency of missed diagnoses in cohort of 140 autopsies not reviewed by a medical renal pathologist

\begin{tabular}{lcc}
\hline Diagnosis & $\begin{array}{c}\text { Total } \\
\text { diagnoses }\end{array}$ & $\begin{array}{c}\text { Missed } \\
\text { diagnoses }\end{array}$ \\
\hline $\begin{array}{l}\text { Diabetic nephropathy } \\
\text { Bile cast nephropathy }\end{array}$ & 22 & 11 \\
Thrombotic & 7 & 1 \\
microangiopathy & 5 & 3 \\
Infection-related GN & 4 & 3 \\
Oxalate nephropathy & 3 & 3 \\
Light-chain cast nephropathy & 2 & 2 \\
Amyloid nephropathy & 2 & 1 \\
Urate nephropathy & 2 & 1 \\
Hemosiderosis & 2 & 1 \\
Focal necrotizing/crescentic & 2 & 0 \\
GN & 1 & 1 \\
Polyoma virus nephropathy & 1 & 1 \\
Vasculitis (ANCA- & & 0 \\
associated) & 1 & \\
Atheroembolic disease & 1 & \\
Nephrocalcinosis & & \\
\hline
\end{tabular}

Abbreviations: ANCA, anti-neutrophil cytoplasmic antibody; GN, glomerulonephritis.

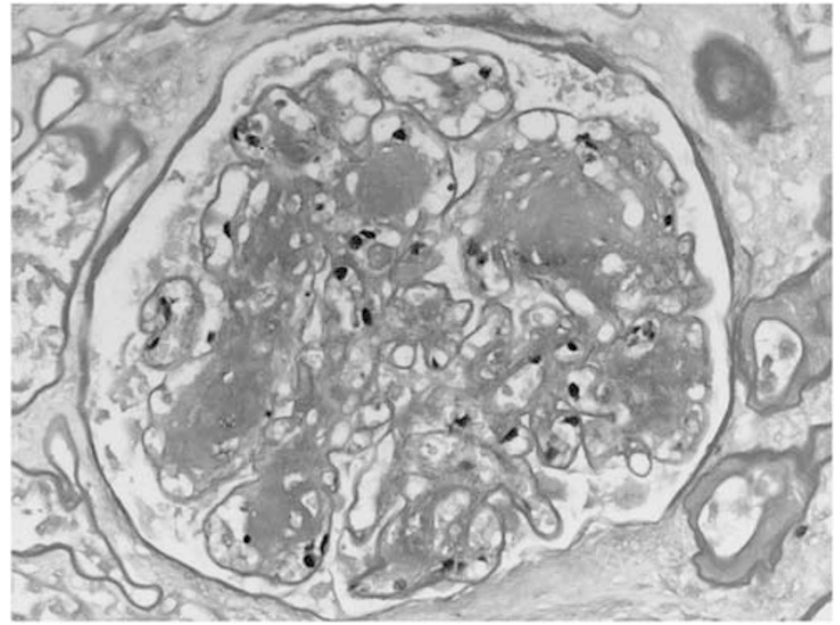

Figure 1 Severe diabetic glomerulosclerosis with KimmelstielWilson nodules and thickened glomerular basement membranes, easily recognizable despite extensive autolytic changes (periodic acid-Schiff, original magnification $\mathrm{x} 400$ ). A full color version of this figure is available at the Modern Pathology journal online.

cells. This patient had a history of peripheral vascular disease and abdominal aortic aneurysm, but there were no documented interventional procedures before death.

Bile cast nephropathy was the second most common diagnosis in our cohort, identified in seven $(5 \%)$ cases. A distinct form of renal tubular injury in patients with severe liver disease, accumulation of bile casts results in direct bile and bilirubin toxicity and tubular obstruction. ${ }^{16}$ Five patients had cirrhosis secondary to either alcohol abuse (2), hepatitis C infection (1), primary sclerosing cholangitis (1), or IgG4-related sclerosing cholangitis (1). The other two patients had acute multiorgan failure following hematopoietic stem cell transplant for hematologic malignancies. All cases were characterized by numerous green-yellow to orange-brown intratubular casts predominantly involving the distal nephron segments in the medulla. Immunohistochemical stains for myoglobin and hemoglobin were negative, excluding other underlying causes of 'pigment nephropathy' (i.e. rhabdomyolysis and hemolysis).

Features of acute thrombotic microangiopathy were identified in 5 (4\%) cases, including 3 patients with hematologic malignancies status-post chemoradiation therapy and hematopoietic stem cell transplant, 1 patient with systemic lupus erythematosus and clinically apparent coagulopathy, and 1 patient with influenza and acute respiratory distress syndrome. These cases were characterized by numerous fibrin-platelet thrombi causing distention and occlusion of the glomeruli capillaries and arterioles, and mucoid intimal expansion of the small arteries with incorporated schistocytes (Figure 2). In addition, one of the hematopoietic stem cell transplant patients had polyomavirus nephropathy with basophilic ground-glass nuclear inclusions in the tubular 


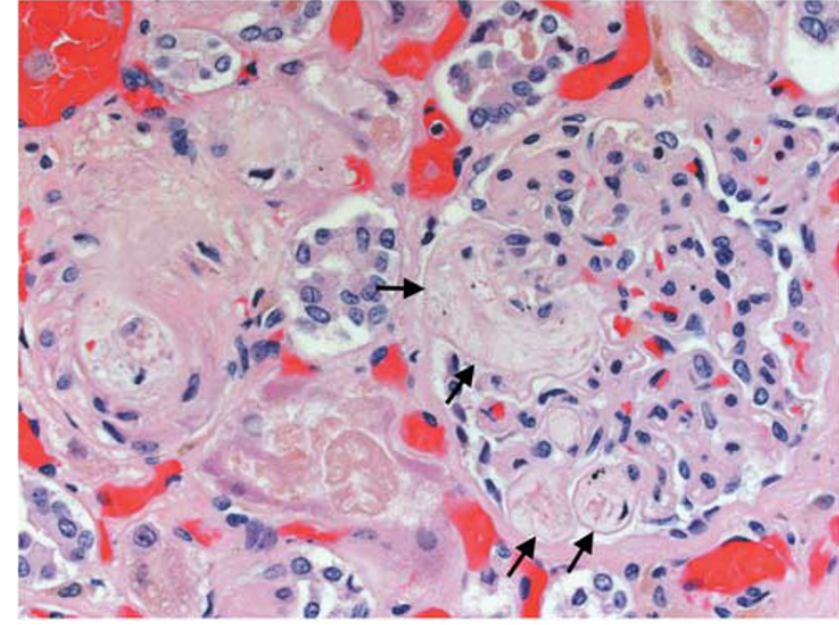

Figure 2 Acute thrombotic microangiopathy shows distention of the glomerular capillaries by fibrin-platelet thrombi and fragmented red blood cells (arrows), and mucoid intimal expansion of an adjacent small artery (hematoxylin and eosin, original magnification $\mathrm{x} 400$ ).

epithelial cells, confirmed by positive immunostaining for SV40 large T antigen.

We identified 4 cases of infection-related glomerulonephritis, 2 of which were patients with endocarditis and 2 with hepatitis $\mathrm{C}$ infection and sepsis. One case of endocarditis-associated glomerulonephritis, which manifested as a focal crescentic glomerulonephritis, was initially identified, but the other 3 diagnoses were missed. The glomeruli in these 3 cases showed a membranoproliferative pattern of injury with enlargement and lobulation of the glomerular capillary tufts, mesangial and occasional endocapillary hypercellularity, and thickening and/or duplication of the glomerular basement membranes (Figure 3a). Immunofluorescence microscopy was performed on the formalin-fixed, paraffinembedded tissue in these cases, confirming immune complex deposition with granular capillary wall and mesangial staining for predominantly IgG (Figure 3b).

There were two cases of focal necrotizing and crescentic glomerulonephritis of the pauci-immune type, which had been identified by the primary pathologist. One patient had been diagnosed with pauci-immune crescentic glomerulonephritis on kidney biopsy 2 weeks before autopsy (Figure 4a). The other patient had a history of type 1 diabetes and but no known glomerular disease. The status of serum ANCAs in both patients was unknown. A small-vessel vasculitis was identified in one additional case, characterized by transmural and perivascular lymphoplasmacytic interstitial inflammation involving the intralobular and arcuate arteries (Figure $4 \mathrm{~b}$ ). The patient had been seen at an outside hospital $\sim 1$ week before death for rash and acute kidney injury, and work-up had revealed a strongly positive p-ANCA titer. There was no evidence of glomerulonephritis in this case.
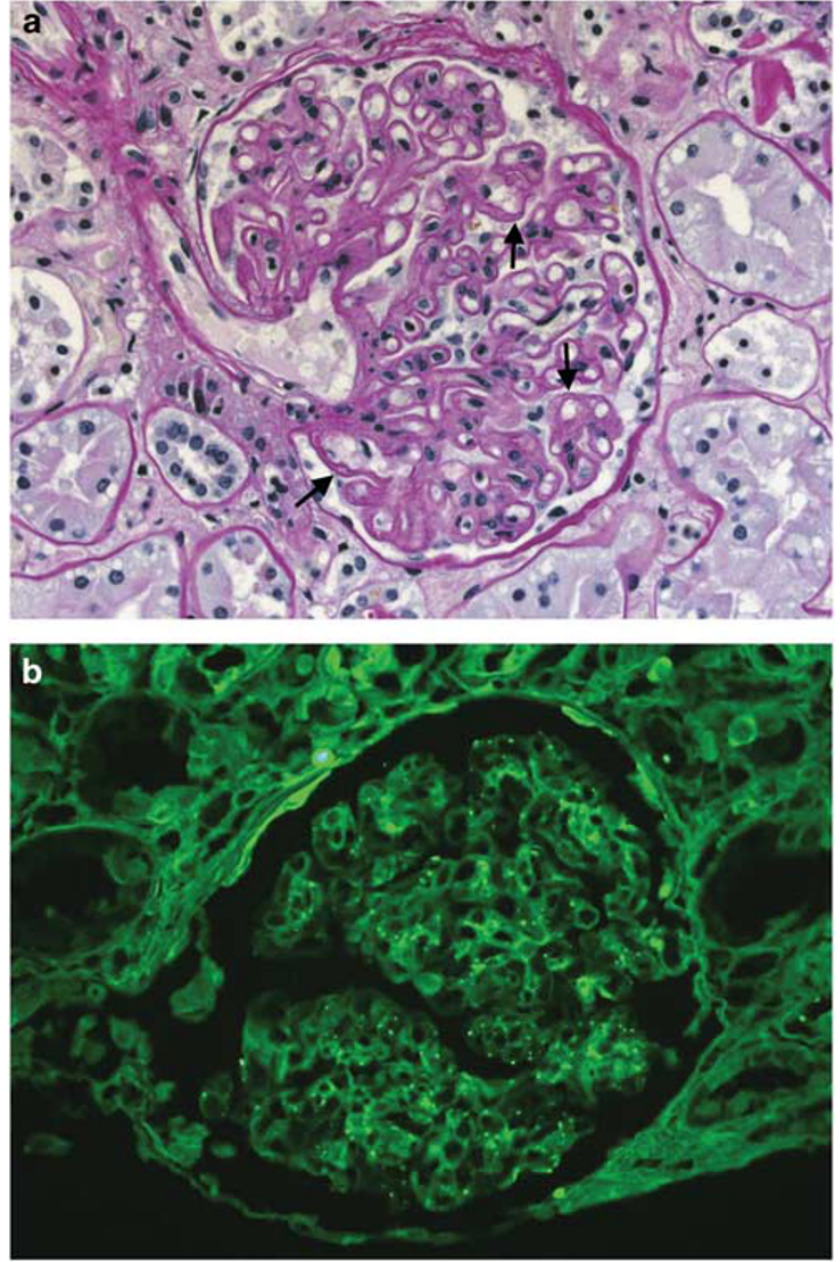

Figure 3 Infection-related glomerulonephritis in a patient with hepatitis C. (a) Segmental endocapillary hypercellularity and duplication (arrows) of the glomerular basement membranes (periodic acid-Schiff, original magnification x400). (b) Granular capillary wall and mesangial staining for IgG by direct immunofluorescence (original magnification x400).

Kidney diseases associated with underlying hematologic malignancy/paraproteinemia were identified in four patients. Light-chain cast nephropathy, also known as 'myeloma cast nephropathy' or 'myeloma kidney,' was present in two cases. One patient had a history of plasma cell myeloma status-post hematopoietic stem cell transplant, and the other patient had untreated chronic lymphocytic leukemia. These cases were characterized by atypical intratubular casts with sharp edges and occasional 'fractures,' predominantly involving the distal nephron segments in the medulla (Figure 5). Immunofluorescence microscopy in both cases showed strong staining of the atypical casts for $\lambda$ light-chain only, confirming their monoclonal nature. We also identified two cases of amyloid nephropathy in patients with plasma cell myeloma. Amorphous eosinophilic material was deposited predominantly in the arterial and arteriolar walls, and was focally seen expanding 

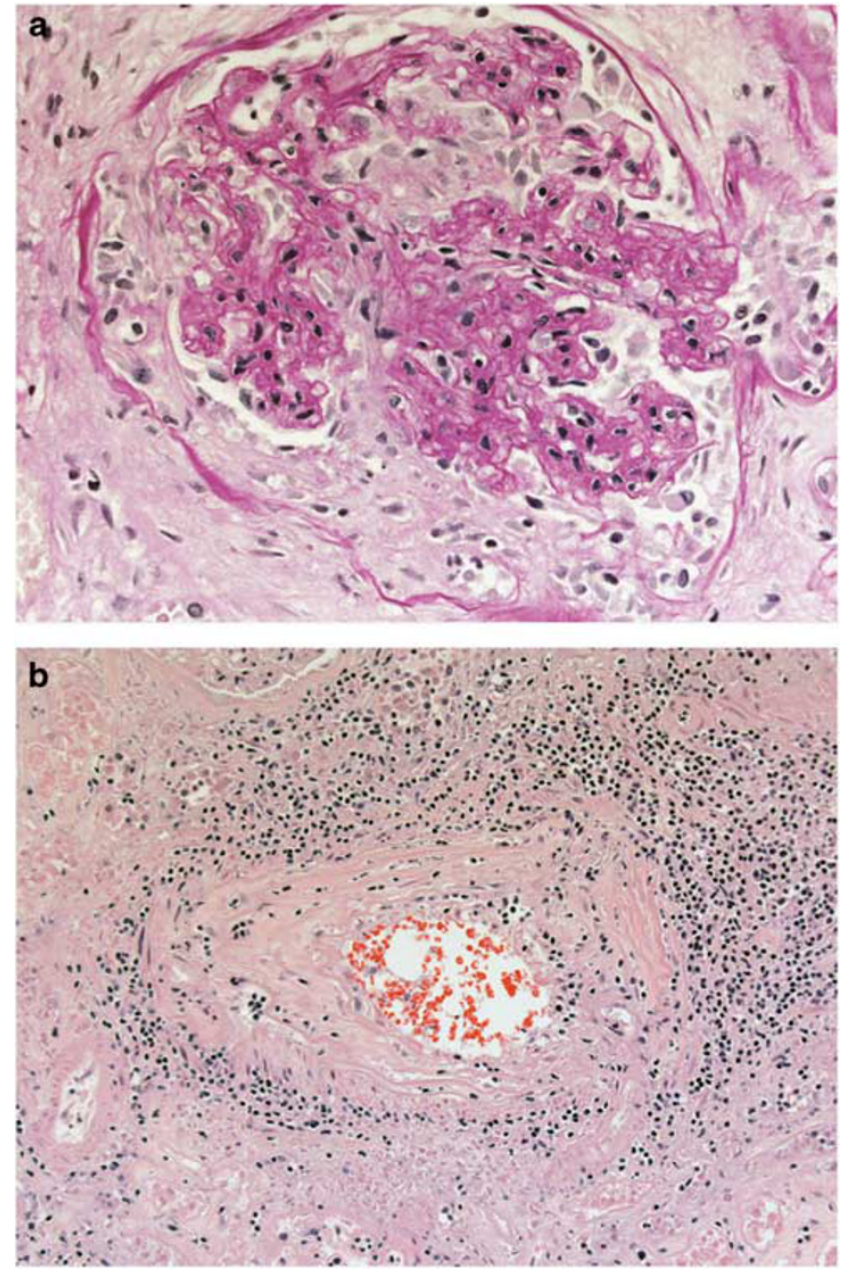

Figure 4 (a) Crescent formation causing disruption of the glomerular capillary tuft and Bowman capsule in a patient with pauci-immune crescentic glomerulonephritis (periodic acidSchiff, original magnification $\mathrm{x} 400$ ). (b) Small vessel vasculitis associated with antineutrophil cytoplasmic antibodies shows transmural and perivascular inflammation involving an arcuate artery (hematoxylin and eosin, original magnification x200).

the glomerular mesangial areas. Congo red stains demonstrated apple green birefringence on polarization microscopy, confirming the diagnosis of amyloidosis.

Several toxic/metabolic tubulointerstitial diseases characterized by deposition of crystals or other injurious substances were also identified. Oxalate nephropathy was present in three cases, demonstrating abundant intratubular translucent crystals, which were birefringent on polarization microscopy. Two of these patients had a history of gastric bypass surgery, a known risk factor for oxalate nephropathy. ${ }^{33}$ The other patient was a type 1 diabetic with chronic kidney disease. Urate nephropathy was present in two patients with gout, showing large collections of needle-shaped uric acid crystals in the medulla. Nephrocalcinosis was correctly identified in one patient with hypertension, diabetes, and chronic kidney disease. Hemosiderosis,

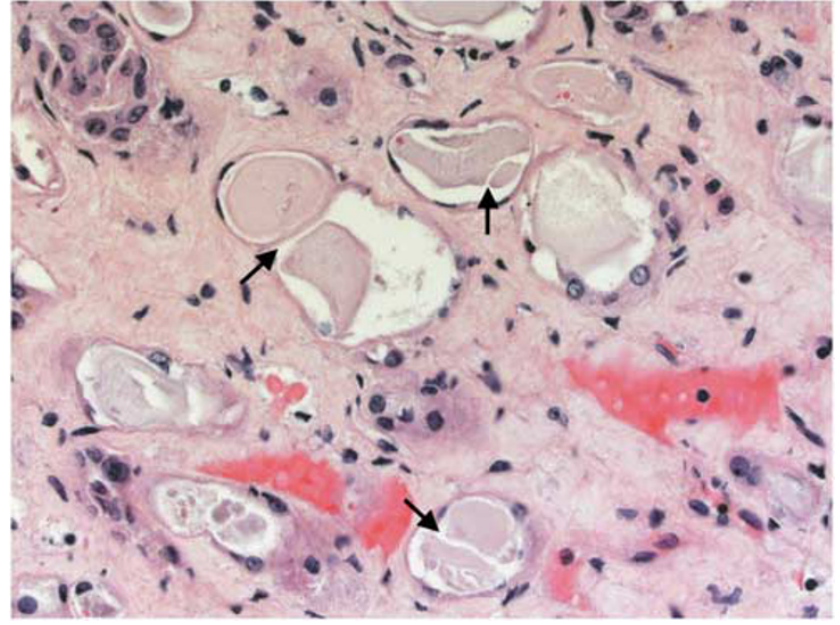

Figure 5 Light-chain cast nephropathy characterized by atypical casts with sharp edges and occasional 'fractures' (arrows) in the distal nephron segments (hematoxylin and eosin, original magnification $\mathrm{x} 200$ ).

Table 3 Full spectrum and frequency of medical renal pathology in 205 total autopsies

\begin{tabular}{lc}
\hline Diagnosis & $\begin{array}{c}\text { Number of } \\
\text { cases }\end{array}$ \\
\hline Diabetic nephropathy & $40(20 \%)$ \\
Bile cast nephropathy & $12(6 \%)$ \\
Thrombotic microangiopathy & $10(5 \%)$ \\
Infection-related GN & $4(2 \%)$ \\
Oxalate nephropathy & $3(1.5 \%)$ \\
Focal necrotizing/crescentic GN & $2(1 \%)$ \\
Amyloid nephropathy & $2(1 \%)$ \\
Nephrocalcinosis & $2(1 \%)$ \\
Light-chain cast nephropathy & $2(1 \%)$ \\
Hemosiderosis & $2(1 \%)$ \\
Urate nephropathy & $2(1 \%)$ \\
Focal segmental glomerulosclerosis & $1(0.5 \%)$ \\
Membranous nephropathy & $1(0.5 \%)$ \\
Rhabdomyolysis-associated tubular injury & $1(0.5 \%)$ \\
with myoglobin casts & \\
Sickle cell nephropathy & $1(0.5 \%)$ \\
Vasculitis (ANCA-associated) & $1(0.5 \%)$ \\
Atheroembolic disease & $1(0.5 \%)$ \\
Polyoma virus nephropathy & $1(0.5 \%)$ \\
\hline
\end{tabular}

Abbreviations: ANCA, anti-neutrophil cytoplasmic antibody; GN, glomerulonephritis.

or tubular accumulation of hemosiderin pigment, was present in two patients with intravascular hemsolysis presumed secondary to cardiac surgical hardware and confirmed in both cases by a positive Prussian blue reaction.

To determine the full spectrum of medical renal disease present in our autopsy cohort, we consolidated the diagnostic findings in these 140 reviewed cases with the 65 autopsy kidneys that had been originally reviewed by a fellowship-trained renal pathologist. Of 205 adult autopsies in a 2-year span, significant medical renal diseases were present in 71 $(35 \%)$ cases, summarized in Table 3. 


\section{Discussion}

This is the first study to comprehensively describe the frequency and spectrum of medical renal diseases in adult autopsy specimens. Since the kidney biopsy procedure is usually avoided in critically ill patients, examination of autopsy kidneys may be the first and only opportunity to identify these diseases. Histologic analysis of the kidneys at autopsy is imperative, as gross examination of the kidneys yields minimal diagnostic information beyond anatomic abnormalities and cystic diseases. ${ }^{34}$ We found a wide variety of significant medical renal pathology in over onethird of adult autopsies in a 2-year span. Diabetic nephropathy was the most common diagnosis in our cohort, which is not surprising given that diabetic nephropathy is the leading cause of chronic kidney disease and end-stage renal disease. ${ }^{3}$ Other frequent diagnoses included thrombotic microangiopathy, glomerulonephritis (frequently infection-related), renal diseases related to underlying hematologic malignancies, and toxic/metabolic tubulointerstitial diseases including bile cast nephropathy and crystal deposition diseases. Accurate identification and reporting of kidney diseases at autopsy is critical as these findings may have implications for the surviving family members, particularly those diseases with a genetic component.

Although there are numerous studies describing clinicopathologic discrepancies at autopsy in terms of antemortem versus post-mortem diagnoses, ${ }^{35,36}$ there is a paucity of literature regarding diagnostic errors committed by pathologists during autopsy evaluation. This is the first study to highlight diagnostic errors of medical renal diseases in autopsy specimens, findings that likely translate to other organ systems. There are several reasons why a surgical pathologist might commit a diagnostic error by overlooking a disease process at autopsy. The primary emphasis during autopsy examination is identification of the immediate cause of death; the heart, lungs, and brain may undergo a more thorough examination as opposed to other organs systems that are not as frequently involved in devastating events. Surgical pathology practices are becoming increasingly subspecialized, so pathologists are also likely to focus on their organ system(s) of expertise. We also speculate that the pathologist may not be adequately trained to recognize medical diseases in autopsy kidney specimens. It has previously been reported that non-neoplastic renal diseases are often missed in tumor nephrectomy specimens. ${ }^{26-30}$ This was thought to be a consequence of primary focus on the tumor and inadequate training in renal pathology during residency; a survey conducted at that time showed that only $36 \%$ of pathology residency programs had a mandatory requirement in medical renal pathology. To address this deficiency, the Accreditation Council for Graduate Medical Education (ACGME) included the requirement of renal pathology in the anatomic pathology curriculum for pathology residents effective from 1 July 2015, which may provide one solution for this knowledge gap. Our study establishes autopsy kidneys as a valuable teaching tool to reinforce the assessment and accurate identification of medical renal pathology throughout the course of residency training. Based on our own departmental experience, we advocate a dedicated renal pathology rotation early in residency training, followed by reinforcement over subsequent years through one-on-one review of autopsy kidneys and non-neoplastic parenchyma in tumor nephrectomies with renal pathologists. Since autopsy kidneys are such a rich source of renal pathology, they can easily be incorporated into didactic lectures and unknown sessions, particularly in residency programs with fewer medical renal biopsies.

Patients with acute and chronic kidney diseases encompass a broad spectrum of medical diseases that are often complex and multisystem in nature, making it difficult to determine the precise contribution of kidney disease to overall mortality. We are aware of only one autopsy study to document renal disease as a major cause of death, accounting for 6 cases $(2.2 \%)$ in their autopsy cohort of 270 patients. ${ }^{24}$ These cases included two patients with sepsis and pyelonephritis, two patients with hydronephrosis, and two patients with polycystic kidney disease. We speculate that kidney disease contributes to mortality in a larger percentage of patients than is currently appreciated. It is natural to draw an association between kidney disease and death in patients with florid acute diseases, such as rapidly progressive (crescentic) glomerulonephritis, or in patients with symptomatic end-stage renal disease either with or without renal replacement therapy. However, it has been established that chronic kidney disease of any etiology substantially increases the risk of cardiovascular disease and death, as well as predisposes to infectious complications and metabolic diseases. ${ }^{37}$ In defining causes of death at autopsy, three distinct levels are distinguished: the immediate cause of death (final disease or condition resulting in death), the underlying cause of death (primary underlying disease that initiated the events leading to death), and the contributing causes of death (critical conditions or comorbidities that significantly contributed to death). ${ }^{38}$ Considering that the most frequent immediate causes of death in our autopsy cohort were cardiovascular and infectious diseases, we postulate that further analysis of these cases might reveal that kidney diseases are common underlying or contributing causes of death. Of note, none of the missed diagnoses in our cohort would have been identified as the immediate or underlying cause of death, but many of these kidney diseases represented unrecognized contributing causes of death (e.g. bile and light-chain cast nephropathies, thrombotic microangiopathy, infection-related glomerulonephritis). Our understanding of the pathogenesis and evolution of kidney 
disease will improve with accurate recognition and reporting of kidney diseases at autopsy.

There are certain challenges to assessment of the kidneys at autopsy, particularly given the desire to minimize costs, and no standard guidelines have been established for their proper evaluation. Two recent reviews provide suggested approaches to autopsy renal pathology including gross examination, tissue allocation, and ancillary studies. ${ }^{39,40} \mathrm{We}$ would emphasize the importance of systematic evaluation of all four compartments of renal parenchyma on $\mathrm{H} \& \mathrm{E}$ stain, namely the glomeruli, tubules, interstitium, and vessels. Thorough examination of both the cortex and medulla is imperative, as some disease processes can be very focal, or only involve a specific region of the kidney. Attention should be given to the identification of abnormal histologic features such as alterations in glomerular matrix and/or cellularity, atypical or pigmented casts, crystals, thrombi, tubulointerstitial and/or vascular inflammation, or deposition of amorphous material. Although most of these lesions can be identified or strongly suspected based on H\&E staining alone, additional histochemical staining can be performed as necessary including PAS, Jones methenamine silver, and/or Congo red stains. If there is a known history or clinical suspicion of medical renal disease, tissue may be saved upfront in the appropriate fixatives for immunofluorescence and/or electron microscopy, a step that may be too time consuming and costly to perform routinely. Notably, both immunofluorescence and electron microscopy can be performed on formalin-fixed, paraffinembedded tissue, although there is some decreased sensitivity in the former and processing artifact in the latter. ${ }^{31,41}$ Findings of uncertain significance should prompt the primary autopsy pathologist to consult with a fellowship-trained renal pathologist, if possible. This occurred with $21 \%$ of the cases in our cohort of 205 autopsies, and most likely resulted in an overall reduced rate of missed diagnoses.

By establishing a baseline of medical renal disease in autopsy kidney specimens, our study highlights that awareness of systemic diseases and past medical history can help guide evaluation of the kidney. A history of diabetes mellitus should prompt PAS staining to determine if there are alterations in glomerular matrix. Of interest, our study demonstrated that advanced diabetic nephropathy was easily identified by surgical pathologists, whereas mild to moderate stages were universally overlooked. In patients with paraproteinemia and/or hematologic malignancy, histologic examination should focus on glomerular alterations, abnormal intratubular casts, and amorphous deposits in any compartment; Congo red stains and direct immunofluorescence or immunohistochemistry for $\kappa$ and $\lambda$ light chains should be obtained. Patients with localized and/or systemic infections may manifest with infection-related glomerulonephritis, showing endocapillary hypercellularity and/or crescents, or tubulointerstitial nephritis in the context of direct parenchymal infection. Findings of acute thrombotic microangiopathy, namely thrombi and/or fibrinoid change in the glomerular capillaries and arterioles, were commonly seen in patients who had undergone hematopoietic stem cell transplant, as has been documented previously. ${ }^{5-6,42,43}$ Similar findings can also be seen in other common clinical scenarios at autopsy including chemoradiation therapy, hemolytic uremic syndrome (classic or atypical), thrombotic thrombocytopenic purpura, malignant hypertension, and disseminated intravascular coagulation. Of note, a history of cardiovascular disease should prompt careful evaluation of the vasculature and the extent of glomerular and tubulointerstitial scarring. For the purposes of this study, we did not consider cases with descriptive findings of arterionephrosclerosis (i.e. global glomerulosclerosis, interstitial fibrosis, tubular atrophy, and arteriosclerosis) as diagnostic entities due to the difficulty of establishing whether a diagnostic discrepancy existed. We also disregarded another disease process, acute tubular injury (or 'acute tubular necrosis'), because of difficulty differentiating the histologic changes from post-mortem autolysis in the absence of clinical correlation. ${ }^{32}$ Finally, we caution against overuse of the term 'autolysis' in the diagnostic line for autopsy kidneys. Of the 23 kidney specimens in our cohort which were dismissed as autolyzed in the original autopsy report, we determined that $96 \%$ had histology sufficient for interpretation and $17 \%$ had significant missed diagnoses. We suspect that the overreliance on autolysis translates to other organ systems.

In summary, our study shows that over one-third of adult autopsies harbor significant renal pathology, encompassing a wide spectrum of disease processes. This study highlights a knowledge gap that is pertinent to all practicing surgical pathologists, who should be aware that medical renal disease is common in adult autopsy kidney specimens but often overlooked. The deficiency in knowledge regarding renal pathology is likely due to limited exposure and current training practices in pathology residency programs. The autopsy has long been recognized for providing important contributions in medical education and quality improvement of care, and we have demonstrated that autopsy kidney specimens are a valuable source of material to supplement training in anatomic pathology and help satisfy the ACGME requirement for renal pathology training. Perhaps, most importantly, recognition of kidney disease and its contribution to death at autopsy will provide practical information about the pathogenesis and critical events during the evolution of kidney disease, leading to a better understanding of disease progression and ultimately improved patient care. 


\section{Disclosure/conflict of interest}

The authors declare no conflict of interest.

\section{References}

$1 \mathrm{Xu}$ J, Murphy SL, Kochanek KD, et al. Deaths: final data for 2013. Natl Vital Stat Rep 2016;64:1-119.

2 Go AS, Chertow GM, Fan D, et al. Chronic kidney disease and the risks of death, cardiovascular events, and hospitalization. N Engl J Med 2004;351:1296-1305.

3 Saran R, Li Y, Robinson B, et al. US Renal Data System 2015 Annual Data Report: epidemiology of kidney disease in the United States. Am J Kidney Dis 2016;67: $\mathrm{S} 1-\mathrm{S} 434$

4 Wang HE, Muntner P, Chertow GM, et al. Acute kidney injury and mortality in hospitalized patients. Am J Nephrol 2012;35:349-355.

5 Brinkerhoff BT, Houghton DC, Troxell ML. Renal pathology in hematopoietic cell transplant recipients: a contemporary biopsy, nephrectomy, and autopsy series. Mod Pathol 2016;29:637-652.

6 El-Seisi S, Gupta R, Clase CM, et al. Renal pathology at autopsy in patients who died after hematopoietic stem cell transplantation. Biol Blood Marrow Transplant 2003;9:683-688.

7 Wyatt CM, Morgello S, Katz-Malamed R, et al. The spectrum of kidney disease in patients with AIDS in the era of antiretroviral therapy. Kidney Int 2009;75: 428-434.

8 Nicolau Laparra MC, Martinez F, Giner V, et al. Temporal evolution of renal involvement in a necropsy study of HIV patients from the pre- and HAART eras. Nefrologia 2010;30:420-426.

9 Monga G, Mazzucco G, Boldorini R, et al. Renal changes in patients with acquired immunodeficiency syndrome: a post-mortem study on an unselected population in northwestern Italy. Mod Pathol 1997;10: 159-167.

10 Shahinian V, Rajaraman S, Borucki M, et al. Prevalence of HIV-associated nephropathy in autopsies of HIVinfected patients. Am J Kidney Dis 2000;35:884-888.

11 Herrera GA, Joseph L, Gu X, et al. Renal pathologic spectrum in an autopsy series of patients with plasma cell dyscrasia. Arch Pathol Lab Med 2004;128:875-879.

12 Ivanyi B.. Frequency of light chain deposition nephropathy relative to renal amyloidosis and Bence Jones cast nephropathy in a necropsy study of patients with myeloma. Arch Pathol Lab Med 1991;79:517-525.

13 Arase Y, Ikeda K, Murashima N, et al. Glomerulonephritis in autopsy cases with hepatitis $\mathrm{C}$ virus infection. Intern Med 1998;37:836-840.

14 Gopalani A, Ahuja TS. Prevalence of glomerulopathies in autopsies of patients infected with the hepatitis C virus. Am J Med Sci 2001;322:57-60.

15 Klessens CQ, Woutman TD, Veraar KA, et al. An autopsy study suggests that diabetic nephropathy is underdiagnosed. Kidney Int 2016;90:149-156.

16 Van Slambrouck CM, Salem F, Meehan SM, et al. Bile cast nephropathy is a common pathologic finding for kidney injury associated with severe liver dysfunction. Kidney Int 2013;84:192-197.

17 Buettner M, Toennes SW, Buettner S, et al. Nephropathy in illicit drug abusers: a postmortem analysis. Am J Kidney Dis 2014;63:945-953.
18 Ohashi R, Ishii H, Shimizu A. Morphological spectrum of renal pathology and its correlation to clinical features in patients with disseminated intravascular coagulation: a study involving a series of 21 autopsy cases. Pathol Int 2014;64:443-452.

19 Hara S, Kawano M, Mizushima I, et al. Distribution and components of interstitial inflammation and fibrosis in IgG4-related kidney disease: analysis of autopsy specimens. Hum Pathol 2016;55:164-173.

20 Trostle DC, Bedetti CD, Steen VD, et al. Renal vascular histology and morphometry in systemic sclerosis. A case-control autopsy study. Arthritis Rheum 1988;31: 393-400.

21 Nakayama EE, Ura S, Fleury RN, et al. Renal lesions in leprosy: a retrospective study of 199 autopsies. Am J Kidney Dis 2001;38:26-30.

22 Faria TV, Baptista MA, Burdmann EA, et al. Renal glomerular alterations in patients with cancer: a clinical and immunohistochemical autopsy study. Ren Fail 2010;32:918-922.

23 Niizuma S, Nakamura S, Ishibashi-Ueda $\mathrm{H}$, et al. Kidney function and histological damage in autopsy subjects with myocardial infarction. Ren Fail 2011;33: 847-852.

24 Ueda K, Omae T, Hirota Y, et al. Epidemiological and clinico-pathological study on renal diseases observed in the autopsy cases in Hisayama population, Kyushu Island, Japan. J Chronic Dis 1976;29: 159-173.

25 Usta U, Tastekin E, Isler E, et al. Histopathological and immune alterations in autopsied kidneys. Saudi Med J 2014;35:1331-1338.

26 Henriksen KJ, Meehan SM, Chang A. Non-neoplastic renal diseases are often unrecognized in adult tumor nephrectomy specimens: a review of 246 cases. Am J Surg Pathol 2007;31:1703-1708.

27 Bijol V, Mendez GP, Hurwitz S, et al. Evaluation of the nonneoplastic pathology in tumor nephrectomy specimens: predicting the risk of progressive renal failure. Am J Surg Pathol 2006;30:575-584.

28 Salvatore SP, Cha EK, Rosoff JS, et al. Nonneoplastic renal cortical scarring at tumor nephrectomy predicts decline in kidney function. Arch Pathol Lab Med 2013;137:531-540.

29 Henriksen KJ, Meehan SM, Chang A. Nonneoplastic kidney diseases in adult tumor nephrectomy specimens: common, harmful, yet underappreciated. Arch Pathol Lab Med 2009;133:1012-1025.

30 Bijol V, Batal I. Non-neoplastic pathology in tumor nephrectomy specimens. Surg Pathol Clin 2014;7: 291-305.

31 Nasr SH, Galgano SJ, Markowitz GS, et al. Immunofluorescence on pronase-digested paraffin sections: a valuable salvage technique for renal biopsies. Kidney Int 2006;70:2148-2151.

32 Kocovski L, Duflou J. Can renal acute tubular necrosis be differentiated from autolysis at autopsy? J Forensic Sci 2009;54:439-442.

33 Nasr SH, D'Agati VD, Said SM, et al. Oxalate nephropathy complicating Roux-en-Y Gastric Bypass: an underrecognized cause of irreversible renal failure. Clin J Am Soc Nephrol 2008;3:1676-1683.

34 Bernardi FD, Saldiva PH, Mauad T. Histological examination has a major impact on macroscopic necropsy diagnoses. J Clin Pathol 2005;58:1261-1264.

35 Roulson J, Benbow EW, Hasleton PS. Discrepancies between clinical and autopsy diagnosis and the value 
of post mortem histology; a meta-analysis and review. Histopathology 2005;47:551-559.

36 Shojania KG, Burton EC, McDonald KM, et al. Changes in rates of autopsy-detected diagnostic errors over time: a systematic review. JAMA 2003;289:2849-2856.

37 Thomas R, Kanso A, Sedor JR. Chronic kidney disease and its complications. Prim Care 2008;35: 329-344.

38 Nichols L, Saunders R, Knollmann FD. Causes of death of patients with lung cancer. Arch Pathol Lab Med 2012;136:1552-1557.

39 Henriksen KJ. Assessment of kidneys in adult autopsies. Diag Histopathol 2017;23:117-125.
40 Paueksakon P, Fogo AB. Autopsy renal pathology. Surg Pathol Clin 2014;7:321-355.

41 Nasr SH, Markowitz GS, Valeri AM, et al. Thin basement membrane nephropathy cannot be diagnosed reliably in deparaffinized, formalin-fixed tissue. Nephrol Dial Transplant 2007;22:1228-1232.

42 Chang A, Hingorani S, Kowalewska J, et al. Spectrum of renal pathology in hematopoietic cell transplantation: a series of 20 patients and review of the literature. Clin J Am Soc Nephrol 2007;2:1014-1023.

43 Troxell ML, Higgins JP, Kambham N. Renal pathology associated with hematopoietic stem cell transplantation. Adv Anat Pathol 2014;5:330-340. 